Journal of Animal and Veterinary Advances 11 (11): 1921-1924, 2012

ISSN: $1680-5593$

(C) Medwell Journals, 2012

\title{
The Effects of Resveratrol Supplementation on Exercise-Induced Apoptosis of Rat Hepatocytes
}

\author{
Shan-Shan Li, Zi-Chao Chen and Chao-Hui Zhang \\ PE Department of Sichuan University, 610064 Chengdu, Sichuan Province, China
}

\begin{abstract}
The present study was designed to determine the effects of resveratrol supplementation on exerciseinduced apoptosis of rat hepatocytes. The rats were divided randomly into the sedentary group (Group I) and exhaustive exercise groups (Group I-IV). Each group contains 12 animals. The rats of exhaustive exercise groups received daily oral administration of resveratrol at doses of 0,200 or $400 \mathrm{mg} / \mathrm{kg} /$ day for 30 days. The rats of sedentary group received daily oral administration of vehicle only $(10 \mathrm{~mL} / \mathrm{kg} /$ day $)$ for the same period. Exhaustive exercise was performed on a motor-driven rodent treadmill. Paraffin-embedded liver sections were stained using the Streptavidin Peroxidase (SP) immunohistochemistry technique for BAX and Bcl-2 detection. The results showed that exhausting exercises could make the expression of BAX proteins in the liver and $\mathrm{BAX} / \mathrm{Bcl}-2$ ratio dramatically increases, the expression of $\mathrm{Bcl}-2$ proteins obviously decreases. After resveratrol supplementation, the expression of $\mathrm{BAX}$ proteins and $\mathrm{BAX} / \mathrm{Bcl}-2$ ratio obviously decreased, the expression of Bcl-2 proteins obviously increased. Which indicated that resveratrol supplementation can inhibit exerciseinduced apoptosis of rat hepatocytes.
\end{abstract}

Key words: Resveratrol, exercise, apoptosis, rat, hepatocytes, SP

\section{INTRODUCTION}

Apoptosis or programmed cell death is a normal physiological function essential for the homeostasis of immuno haemopoietic tissues. This process occurs via specific signaling pathways, eventually leading to DNA fragmentation, nuclear condensation, proteolysis and cell fragmentation (Reed, 2001; Albert, 2001). Within the past 10 years, apoptosis has gained the interest of many exercise scientists because in addition to necrotic cell death, evidence indicates that apoptotic cell death also occurs with intense exercise. Intense exercise is often accompanied by an excessive generation of oxygen free radicals. Reactive oxygen intermediates react with cellular components which may cause extensive damage to cellular structures such as DNA. The DNA damage could finally lead to apoptotic or necrotic processes in many organs including skeletal muscles, heart, cells of the immune system, lungs and liver (Phaneuf and Leeuwenburgh, 2001; Song et al., 2006; Kavazis et al., 2008).

Resveratrol (3, 4', 5-tri-hydroxystilbene), a poly phenolic stilbene first isolated from the root of Veratrum grandiflorum, occurs naturally in grapes, mulberries and peanuts (Aggarwal et al., 2004; Saiko et al., 2008; Hiroto et al., 2011). In these plants resveratrol is synthesized in response to stress conditions such as an infection and thus can be considered to be a phytoalexin. Resveratrol has estrogenic activity in mammals and therefore is classified as a phytoestrogen (Niles et al., 2006). Recent studies indicate that resveratrol possesses many biological activities that are protective against atheroscrelosis including anti-oxidant, anti-inflammatory, anti-platelet and vasorelaxant activities (Delmas et al., 2005; Xia et al., 2010). In addition, resveratrol is identified as a cancer chemotherapeutic agent and has recently been reported to mimic effects of dietary restriction and extends the lifespans of lower organisms and improves the health and survival of mice on a high-fat diet (Alarcon de la Lastra and Villegas, 2005; Pervaiz and Holme, 2009; Fukui et al., 2010). However, the effects of resveratrol on exercise-induced apoptosis is poorly understood. The purpose of the present study was to investigate the effects of resveratrol supplementation on exerciseinduced apoptosis of rat hepatocytes.

\section{MATERIALS AND METHODS}

Main reagents: Resveratrol (>98\% purity, FW 228.2) were purchased obtained from Cuiyuan Biotechnology Co., Ltd. (Xi'an, China). Rabbit polyclonal antibody against human BAX and Bcl-2 and goat anti-rabbit polyclonal antibody were purchased from Zhongshan Biotechnology Co., Ltd. (Beijing, China). Other chemicals and

Corresponding Author: Zi-Chao Chen, PE Department of Sichuan University, 610064 Chengdu, Sichuan Province, China 
biochemicals were of analytical grade and were purchased from Sigma Chem. Co. (St. Louis, MO, USA) and Changsheng Pharmaceutical Co., (Chengdu, China) unless otherwise indicated.

Animals: Male Wistar rats weighing 140-180 g were obtained from Sichuan Research Animal Center (Chengdu, China). Rats were housed in a room with alternating $12 \mathrm{~h}$ periods of light and dark, ambient temperature of $24 \pm 1^{\circ} \mathrm{C}$ and humidity of $55 \pm 5 \%$. All animals were allowed free access to distilled water and fed on a commercial diet (purchased from Sichuan Research Animal Center). All animals received humane care according to the guideline of Guidebook for the Care and Use of Laboratory Animals. The study protocol was approved by the animal research ethics committee at Sichuan University (Chengdu, China).

Animals grouping and treatment: After being adaptively fed for a week, the rats were divided randomly into the sedentary group (Group I) and exhaustive exercise groups (Group I-IV). Each group contains 12 animals. The rats of exhaustive exercise groups received daily oral (gavage) administration of resveratrol at doses of 0,200 or $400 \mathrm{mg} / \mathrm{kg} /$ day for 30 days. Resveratrol was administered in a vehicle of $0.5 \%(\mathrm{w} / \mathrm{v})$ aqueous methylcellulose containing $0.2 \%(\mathrm{w} / \mathrm{v})$ Tween 80 , a dosing volume of $10 \mathrm{~mL} / \mathrm{kg} /$ day was used. The rats of sedentary group received daily oral (gavage) administration of vehicle only $(10 \mathrm{~mL} / \mathrm{kg} /$ day $)$ for the same period.

Exercise program: After 30 days, the rats of exhaustive exercise groups were exercised vigorously in one session until they were exhausted. The exercise protocol was $22 \mathrm{~m} \mathrm{~min}^{-1}$ at $10 \%$ inclination on a motor-driven rodent treadmill. A rat was considered exhausted when it would not be prompted to run any more. The rats would stop running and when overturned would continue to lie on their backs disregarding gentle prods and electric shock (Chen et al., 2002). Immediately after exhaustive exercise, all the rats were sacrificed by decapitation. The liver were extracted and treated with $4 \%$ paraformaldehyde in $0.1 \mathrm{M}, \mathrm{pH} 7.4$ Phosphate Buffered Saline (PBS) for $24 \mathrm{~h}$ before the embedding in paraffin. The tissues were cut into serial sections of $4 \mu \mathrm{m}$ thickness.

The expression of $\mathrm{BAX}$ and $\mathrm{Bcl}-2$ proteins: Paraffinembedded liver sections were stained using the Streptavidin Peroxidase (SP) immunohistochemistry technique for BAX and Bcl-2 detection (Bai and Meng, 2005; Wang et al., 2007). Briefly, sections (4 $\mu \mathrm{m})$ were incubated with $3 \mathrm{~mL} \mathrm{~L}^{-1}$ hydrogen peroxide in methanol for $30 \mathrm{~min}$ to block endogenous peroxidase activity, washed in Phosphate Buffered Saline (PBS) and incubated in $100 \mathrm{~mL} \mathrm{~L}^{-1}$ normal goat serum for $20 \mathrm{~min}$ to reduce nonspecific antibody binding. Sections were then incubated with rabbit polyclonal antibody at a dilution of 1:100 against human $\mathrm{BAX}$ and $\mathrm{Bcl}-2$ overnight at $4^{\circ} \mathrm{C}$ followed by three washes with PBS then incubated with biotinylated goat anti-rabbit polyclonal antibody at a dilution of 1:100 for $30 \mathrm{~min}$ followed by 3 washes. Slides were treated with streptavidin peroxidase reagent for $30 \mathrm{~min}$ at a dilution of 1:100 and washed 3 times with PBS.

Finally, slides were incubated in PBS containing diaminobenzidine and $10 \mathrm{~L} \mathrm{~L}^{-1}$ hydrogen peroxide for $10 \mathrm{~min}$, counterstained with hematine and mounted. The rabbit antibody was replaced by PBS as a blank (Wang et al., 2007). Five high power fields of each section were selected randomly and input to the HM IAS-2000 analysis system for staining intensity (average absorbance) analysis.

Statistical analysis: The data are expressed as means \pm SD. Statistical comparisons were compared by one-way Analysis of Variance (ANOVA). The results were considered statistically significant if the p-values were 0.05 or less.

\section{RESULTS AND DISCUSSION}

As shown in Fig. 1 and Table 1, the expression of BAX proteins in the Group II and III were significantly increased compared with that in the Group I $(p<0.05)$. Although, the expression of BAX proteins in the Group IV was also increased, no significant difference was observed $(\mathrm{p}>0.05)$. The expression of BAX proteins in the Group III and IV were significantly decreased compared with that in the Group II $(\mathrm{p}<0.05)$.

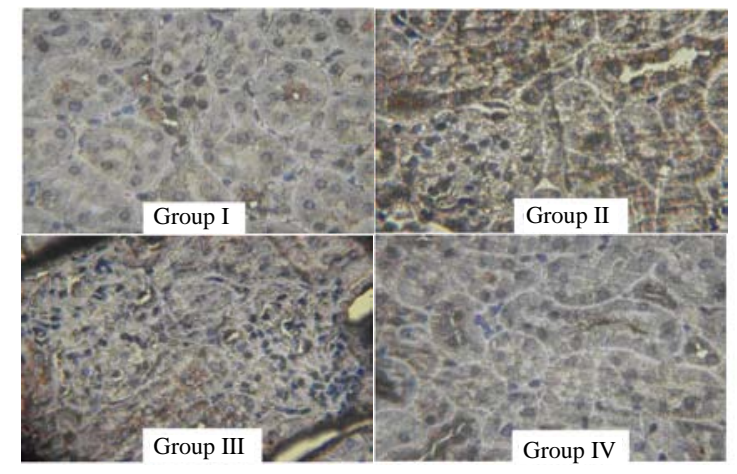

Fig. 1: The expression of BAX proteins in the liver of rats 


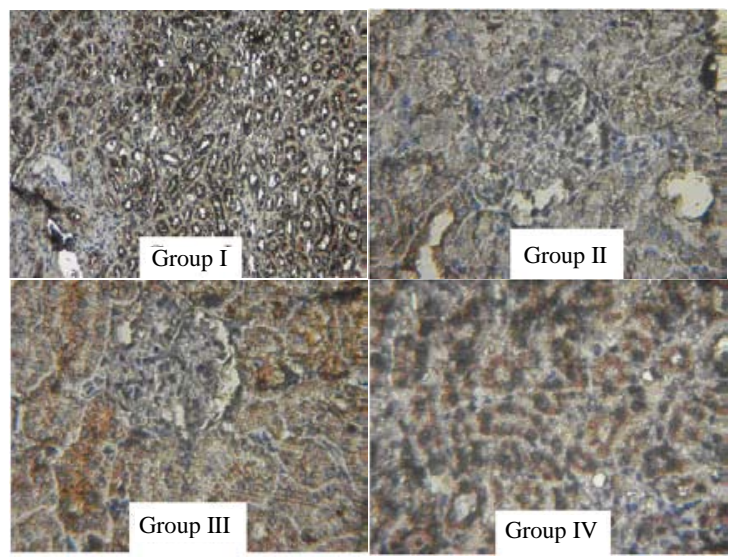

Fig. 2: The expression of Bcl-2 proteins in the liver of rats

$\begin{aligned} & \text { Table 1: } \\
& \text { The comparison of } \mathrm{BAX}, \mathrm{Bcl}-2 \\
& \text { groups }\end{aligned}$
\begin{tabular}{lccc}
\hline Groups & $\mathrm{BAX}$ & $\mathrm{Bcl}-2$ & $\mathrm{BAX} / \mathrm{Bcl}-2$ \\
\hline I & $14.23 \pm 1.17$ & $19.77 \pm 2.14$ & 0.72 \\
II & $22.49 \pm 2.84^{*}$ & $11.64 \pm 1.53^{*}$ & $1.93^{*}$ \\
III & $17.53 \pm 1.69^{* *}$ & $15.83 \pm 1.87^{* * *}$ & $1.11^{* * *}$ \\
IV & $15.15 \pm 2.03^{*}$ & $16.42 \pm 1.75^{* *}$ & $0.92^{* * *}$ \\
\hline
\end{tabular}

Values are mean $\pm \mathrm{SD},{ }^{*} \mathrm{p}<0.05$ compared with Group I. ${ }^{* *} \mathrm{p}<0.05$ compared with Group II

As shown in Fig. 2 and Table 1, the expression of Bcl-2 proteins in the Group II-IV were significantly decreased compared with that in the Group I $(p<0.05)$. The expression of Bcl-2 proteins in the Group III and IV were significantly increased compared with that in the Group II $(\mathrm{p}<0.05)$.

As shown in Table 1, the BAX/Bcl-2 ratio in the Group II-IV were significantly increased compared with that in the Group I $(\mathrm{p}<0.05)$. The BAX/Bcl -2 ratio in the Group III and IV were significantly decreased compared with that in the Group II $(\mathrm{p}<0.05)$. Apoptosis manifests in two major execution programs downstream of the death signal: the caspase pathway and organelle dysfunction of which mitochondrial dysfunction is the best characterized (Gross et al., 1999; Korsmeyer et al., 2000; Gross, 2001). As the Bcl-2 family members reside upstream of irreversible cellular damage and focus much of their efforts at the level of mitochondria, they play a pivotal role in deciding whether a cell will live or die (Fig. 3). $\mathrm{Bcl}-2$ and BAX are two members of the Bcl-2 family which play different roles in programmed cell death (Huckelhoven et al., 2003; Liu et al., 2005). When BAX was overexpressed in cells, apoptotic death in response to death signals was accelerated, leading to its designation as a death agonist and when Bcl-2 was overexpressed it heterodimerized with BAX and death was repressed (Korsmeyer, 1999; Jang et al., 2002). Therefore, the $\mathrm{BAX} / \mathrm{Bcl}-2$ ratio is important in determining susceptibility to apoptosis (Raisova et al., 2001).

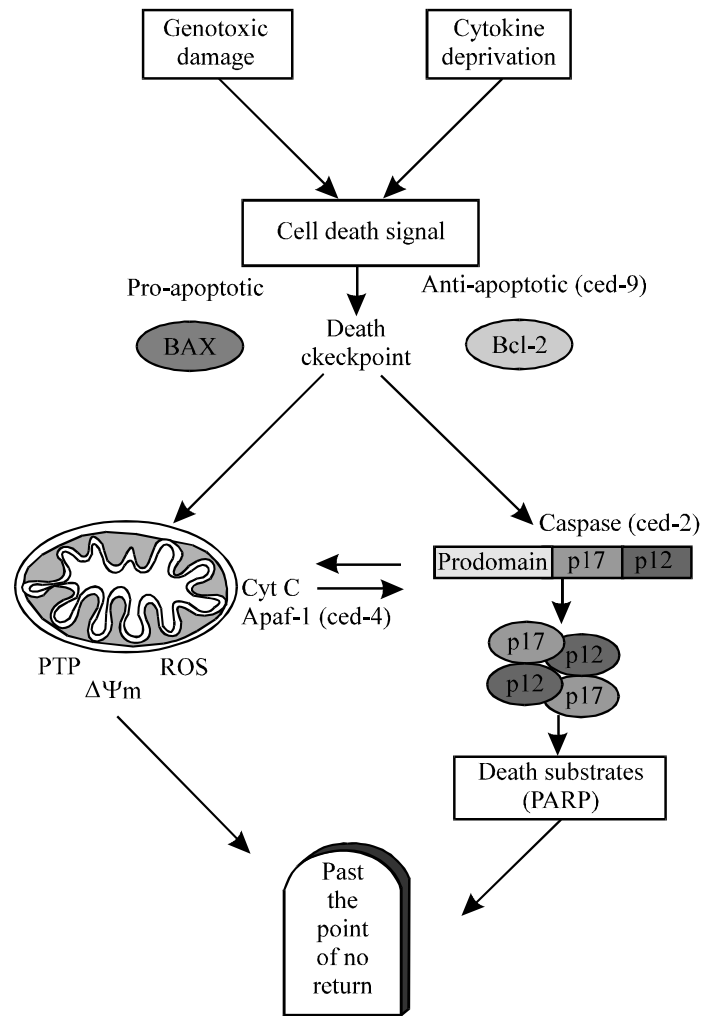

Fig. 3: Schematic Model of mammalian cell death pathway

\section{CONCLUSION}

The data showed that exhausting exercises could make the expression of BAX proteins in the liver and $\mathrm{BAX} / \mathrm{Bcl}-2$ ratio dramatically increases, the expression of Bcl-2 proteins obviously decreases. The results indicated that BAX and Bcl-2 co-regulated the exercise-induced apoptosis of rat hepatocytes.

BAX promotes the apoptosis as up-regulated genes while Bcl-2 inhibits apoptosis as downregulated genes and this may be the gene regulation mechanism of exercise-induced apoptosis. After resveratrol supplementation, the expression of BAX proteins and $\mathrm{BAX} / \mathrm{Bcl}-2$ ratio obviously decreased, the expression of $\mathrm{Bcl}-2$ proteins obviously increased. The results indicated that resveratrol supplementation can inhibit exercise-induced apoptosis of rat hepatocytes.

\section{ACKNOWLEDGEMENT}

This research was supported by the Sichuan University Cross-Disciplinanary Research Projects (No.: SKQY201109). 


\section{REFERENCES}

Aggarwal, B.B., A. Bhardwaj, R.S. Aggarwal, N.P. Seeram, S. Shishodia and Y. Takada, 2004. Role of resveratrol in prevention and therapy of cancer: Preclinical and clinical studies. Anticancer Res., 24: 2783-2840.

Alarcon de la Lastra, C. and I. Villegas, 2005. Resveratrol as an anti-inflammatory and anti-aging agent: Mechanisms and clinical implications. Mol. Nutr. Food Res., 49: 405-430.

Albert, M.L., 2001. Death-defying immunity: Do apoptotic cells influence antigen processing and presentation?. Nat. Rev. Immunol., 4: 223-231.

Bai, J. and Z. Meng, 2005. Expression of apoptosis-related genes in livers from rats exposed to sulfur dioxide. Toxicology, 216: 253-260.

Chen, C.Y., G.I. Holtzman and R.M. Bakhit, 2002. Highgenistin isoflavone supplementation modulated erythrocyte antioxidant enzymes and increased running endurance in rats undergoing one session of exhausting exercise-A pilot study. Pak. J. Nutr., 1: 1-7.

Delmas, D., B. Jannin and N. Latruffe, 2005. Resveratrol: Natural properties against atherosclerosis, associated pro-inflammatory effects and aging. Mol. Nutr. Food Res., 49: 377-395.

Fukui, M., N. Yamabe and B.T. Zhu, 2010. Resveratrol attenuates the anticancer efficacy of paclitaxel in human breast cancer cells in vitro and in vivo. Eur. J. Cancer, 46: 1882-1891.

Gross, A., 2001. BCL-2 proteins: regulators of the mitochondrial apoptotic program. IUBMB Life, 52: 231-236.

Gross, A., J.M. Mc Donnell and S.J. Korsmeyer, 1999. Bcl-2 family members and the mitochondria in apoptosis. Genes Dev., 13: 1899-1911.

Hiroto, Y., K. Tadokoro, T. Tsuda, E. Nakazono and K. Ohnaka et al., 2011. Resveratrol, a phytoestrogen found in red wine, down-regulates protein $\mathrm{S}$ expression in HepG2 cells. Thromb. Res., 127: 1-7.

Huckelhoven, R., C. Dechert and K.H. Kogel, 2003. Overexpression of barley BAX inhibitor 1 induces breakdown of mlo-mediated penetration resistance to Blumeria graminis. Proc. Natl. Acad. Sci., 100: 5555-5560.

Jang, M.H., M.C. Shin, H.S. Shin, K.H. Kim, H.J. Park, E.H. Kim and C.J. Kim, 2002. Alcohol induces apoptosis in TM3 mouse Leydig cells via BAX-dependent caspase-3 activation. Eur. J. Pharmacol., 449: 39-45.
Kavazis, A.N., J.M. McClung, D.A. Hood and S. K. Powers, 2008. Exercise induces a cardiac mitochondrial phenotype that resists apoptotic stimuli. Am. J. Physiol. Heart, 294: H928-H935.

Korsmeyer, S.J., 1999. BCL-2 gene family and the regulation of programmed cell death. Cancer Res., 59: $1693 \mathrm{~s}-1700 \mathrm{~s}$.

Korsmeyer, S.J., M.C. Wei, M. Saito, S. Weiler, K.J. Oh and P.H. Schlesinge, 2000. Pro-apoptotic cascade activates $\mathrm{BD}$, which oligomerizes $\mathrm{BAK}$ or $\mathrm{BAX}$ into pores that result in the release of cytochrome. Cell Death Differ., 7: 1166-1173.

Liu, J.J., R.W. Huang, D.J. Lin, J. Peng and X.Y. Wu et al., 2005. Expression of survivin and $\mathrm{BAX} / \mathrm{Bcl}-2$ in peroxisome proliferator activated receptor-gamma ligands induces apoptosis on human myeloid leukemia cells in vitro. Ann. Oncol., 16: 455-459.

Niles, R.M., C.P. Cook, G.G. Meadows, Y.M. Fu, J.L. McLaughlin and G.O. Rankin, 2006. Resveratrol is rapidly metabolized in athymic $(\mathrm{nu} / \mathrm{nu}) \mathrm{m}$,ice and does not inhibit human melanoma xenograft tumor growth. J. Nutr., 136: 2542-2546.

Pervaiz, S. and A.L. Holme, 2009. Resveratrol: Its biologic targets and functional activity. Antioxid. Redox. Signal., 11: 2851-2897.

Phaneuf, S. and C. Leeuwenburgh, 2001. Apoptosis and exercise. Med. Sci. Sports Exer., 33: 393-396.

Raisova, M., A.M. Hossini, J. Eberle, C. Riebeling and T. Wieder et al., 2001. The Bax/Bcl-2 ratio determines the susceptibility of human melanoma cells to CD95/Fas-mediated apoptosis. J. Invest. Dermatol., 117: 333-340.

Reed, J.C., 2001. Apoptosis-regulating proteins as targets for drug discovery. Trends Mol. Med., 7: 314-319.

Saiko, P., A. Szakmary, W. Jaeger and T. Szekeres, 2008. Resveratrol and its analogs: defense against cancer, coronary disease and neurodegenerative maladies or just a fad? Mutat. Res., 658: 68-94.

Song, W., H.B. Kwak and J.M. Lawler, 2006. Exercise training attenuates age-induced changes in apoptotic signaling in rat skeletal muscle. Antioxid. Redox. Signal., 8: 517-528.

Wang, J.M., B.L. Xiao, J.W. Zheng, H.B. Chen and S.Q. Zou, 2007. Effect of targeted magnetic nanoparticles containing 5-FU on expression of Bcl-2, BAX and caspase 3 in nude mice with transplanted human liver cancer. World J. Gastroenterol., 13: 3171-3175.

Xia, E.Q., G.F. Deng, Y.J. Guo and H.B. Li, 2010. Biological activities of polyphenols from grapes. Int. J. Mol. Sci., 11: 622-646. 